\title{
EFEITOS DE CENÁRIOS DE USO DO SOLO SOBRE O REGIME HIDRÍCO E PRODUÇÃO DE SEDIMENTOS NA BACIA DO RIBEIRÃO CONCÓRDIA - SC ${ }^{1}$
}

\author{
Mauricio Perazzoli², Adilson Pinheiro³ e Vander Kaufmann²
}

\begin{abstract}
RESUMO - O uso inadequado dos solos agrícolas vem causando a perda gradual da sua capacidade produtiva e a degradação dos recursos hídricos por sedimentos e poluentes. O uso do solo pode afetar os processos hidrológicos e a erosão do solo na bacia. O objetivo do trabalho foi simular diferentes cenários de uso do solo para a bacia hidrográfica do ribeirão Concórdia, utilizando o modelo SWAT, e analisar os efeitos dessas mudanças no fluxo de água e de sedimentos. Os cenários gerados foram agricultura, mata nativa e pastagem. A bacia está localizada no sul do Brasil e possui uma área de drenagem de $30,74 \mathrm{~km}^{2}$. Na etapa de calibração, obteve-se coeficente de eficiência Nash-Sutcliffe mensais de 0,82 para escoamento e 0,83 para sedimento. O cenário mata nativa apresentou o maior decréscimo na vazão média, em relação ao cenário atual, cerca de $8,70 \%$. O cenário agricultura produziu a maior taxa de produção de sedimentos, aproximadamente 3 vezes maior que o cenário atual.Observou-se que alterações no uso do solo impactam o regime e a disponibilidade hídrica da bacia.
\end{abstract}

Palavras-chaves: Uso do solo; Modelo SWAT; Regime hídrico.

\section{EFFECTS OF SCENARIOS OF LANDUSE ON WATER REGIME AND SEDIMENT TRANSPORT IN THE CONCÓRDIA RIVER BASIN, SC}

\begin{abstract}
The inappropriate use of agricultural land is causing a gradual loss of its productive capacity and degradation of water resources by sediments and pollutants. Land use can affect the hydrological processes and soil erosion in the basin. The objective of this work was to simulate different scenarios of land use in the Concórdia River basin using the SWAT model, and to analyze the effects of changes in stream flow and sediment yield, making comparisons with the present land use classification. The scenarios generated were agriculture, native forest and pasture. The basin is located in southern Brazil and has a drainage area of $30.74 \mathrm{~km} 2$. In the calibration state, it was obtained the month efficiency coefficient of Nash-Sutcliffe of 0.82 and 0.83 for outflow and sediment yield, respectively. Native forest had the highest decrease in average stream flow, in relation to the present scenario, about $8.70 \%$. Agriculture produced the highest sediment loads, approximately 3 times larger than the present scenario. It was observed that changes in land use can impact the regime and water availability in the basin.
\end{abstract}

Keywords: Land use; SWAT model; Water regime.

\footnotetext{
${ }^{1}$ Recebido em 15.03.2012 aceito para publicação em 13.08.2013.

${ }^{2}$ Programa de Pós-Graduação em Engenharia Ambiental da FURB. Fundação Universidade Regional de Blumenau. E-mail: <mauricio.perazzoli@gmail.com>e <ambitec.amb@gmail.com>.

${ }^{3}$ Departamento de Engenharia Civil da Fundação Universidade Regional de Blumenau. E-mail: <pinheiro@furb.br> .
} 


\section{INTRODUÇÃO}

O ciclo hidrológico interfere em diversos aspectos da vida humana, como a produtividade agrícola, a geração de energia, o controle de cheias, o abastecimento de água para a indústria e para a população, o manejo da flora e da fauna, dentre outros. O mesmo é suscetível às modificações procedentes tanto de causas naturais, como das atividades antrópicas. Conforme descreve Tucci (2002), a ação do homem sobre o uso e ocupação do solo pode produzir impactos nos processos hidrológicos terrestres, como a redução ou aumento da vazão média, máxima e mínima e alterações na qualidade da água de uma bacia hidrográfica.

O uso inadequado dos solos agrícolas vem causando a perda gradual da sua capacidade produtiva e a degradação dos recursos hídricos por sedimentos e poluentes. A ação antrópica tem acelerado esses processos de maneira intensa, trazendo, como consequência, prejuízos ao setor agrícola e ao meio ambiente, com reflexos econômicos e sociais (LELIS; CALIJURI, 2010; PINHEIRO et al., 2011).

Os impactos decorrentes de uso do solo em termos hidrológicos e de transporte de sedimentos afetam o escoamento superficial, a vazão máxima de cheia, os fluxos de base, a recarga subterrânea, umidade do solo e o volume de erosão e sedimentação. Os impactos do uso do solo na qualidade da água referem-se à quantidade de matéria orgânica e nutrientes presente no meio, incremento de organismos patogênicos, pesticidas, metais pesados e alterações no regime termal (FAO, 2000; BONNET et al., 2008).

A resposta hidrológica de uma bacia hidrográfica, a diferentes sistemas de uso e manejo do solo, é importante no processo de modelagem do escoamento superficial. O conhecimento dos efeitos da variação no uso da terra sobre o movimento de sedimentos e água através da bacia hidrográfica é essencial para a tomada de decisões sobre o manejo de uso da terra (PRUSKI et al., 2001; KUHNLE et al., 1996).

Algumas pesquisas envolvendo a utilização de modelos hidrológicos distribuídos vêm sendo realizadosno Brasil, para avaliar os impactos das mudanças de uso e ocupação do solo, no escoamento superficial e na taxa de produção de sedimentos. Com o auxilio de modelos hidrológicos, como o SWAT, são simulados diferentes cenários de usos, para uma mesma bacia hidrográfica. O modelo SWAT (Soil and Water Assessment Tool) foi desenvolvido para verificar os efeitos resultantes das modificações no uso do solo sobre o escoamento superficial e subterrâneo, bem como a produção de sedimentos e na qualidade das águas (NEITSCH et al., 2005).

Em Santa Catarina, três trabalhos foram desenvolvidos visando avaliar o efeito da mudança no uso do solo sobre as vazões e a produção de sedimentos. O primeiro foi realizado por Lino (2009), em uma bacia com floresta Ombrófila Mista. Foi observado que o maior impacto na redução do escoamento superficial e da produção de sedimentos foi obtido pela substituição do uso e cobertura atual do solo pelo cenário mata nativa. O outro trabalho foi desenvolvido por Blainski et al. (2011), em uma bacia com floresta Ombrófila Densa, situado na região sul do Estado. Os resultados demonstraram que a vazão média diária anual foi maior no cenário com solo exposto, sendo esse aumento atribuído à redução da infiltração de água no solo e ao aumento do escoamento superficial. Blainski et al. (2010b) realizaram trabalho semelhante na bacia hidrográfica do Lajeado dos Fragosos, com Floresta Estacional Decidual. Os resultados obtidos demonstraram que o cenário reflorestamento apresentou as menores perdas de solo por erosão hídrica. Em São Paulo, Machado, Vettorazzi e Xavier (2003), aplicaram o modelo SWAT para simulação de cenários de uso do solo na bacia hidrográfica do Ribeirão dos Marins. Eles obtiveram uma redução de 94,0 \% na produção de sedimentos com a substituição da pastagem por vegetação nativa.

Nesse sentido,esse trabalho teve como objetivo simular diferentes cenários de uso do solo para a bacia hidrográfica do ribeirão Concórdia e analisar os efeitos dessas mudanças no fluxo de água e de sedimentos, fazendo-se comparações com a classificação atual de uso do solo.

\section{MATERIAL E MÉTODOS}

A metodologia deste trabalho compreende a aplicação do modelo hidrológico SWAT, integrado com o software de SIG ArcView (ArcSWAT 2009.93.7b), para a simulação de diferentes cenários de usos e ocupação do solo à bacia hidrográfica do ribeirão Concórdia. Os efeitos das mudanças no uso e ocupação do solo são avaliados em termos de regime hídrico e na produção de sedimentos. 


\section{1. Área de estudo}

A bacia hidrográfica do ribeirão Concórdia está localizada no município de Lontras, no estado de Santa Catarina, Brasil. Trata-se de uma pequena bacia rural representativa que possui uma área de drenagem de $30,74 \mathrm{~km}^{2}$.

Na bacia existe uma rede de monitoramento, cuja implantação foi iniciada em 2006. Existem instalados na bacia: oito pluviógrafos, três estações fluviométricas (duas com sensor de nível Thalimedes OTT e uma com vertedor), um turbidimetro DTS 12 (com resolução de 0 a 3000 NTU), além de uma estação meteorológica automática completa. Nas estações fluviométricas são também coletadas, amostras de sedimentos em amostradores de nível ascendente (ANA) e de nível descendente (AND). A instalação e funcionamento destes amostradores são descritos em Paranhos et al. (2002).

Dessa rede de monitoramento foram obtidos os dados meteorológicos e hidrológicos utilizados como entrada para o modelo. Os dados relativos às características físicas e hidrológicas dos solos foram obtidos dos estudos de Teixeira (2008), que analisou o solo em 20 pontos da bacia, coletando amostras em três diferentes profundidades ( 0 a 20, 40 a 60 e 80 a $100 \mathrm{~cm}$ ), em um total de 60 amostras. Deste estudo, foram utilizados os valores da massa específica aparente, teores de argila, silte e areia, condutividade hidráulica saturada e carbono orgânico. No mesmo trabalho, Teixeira (2008) também concluiu que, em relação ao grupo hidrológico, os solos da bacia podem ser enquadrados no grupo C. Outro parâmetro necessário, o albedo foi estimado através da cor do solo. Ele foi determinado por comparação visual utilizando-se a carta de Munsell, através daexpressão (Post et al. 2000):

$$
A l b=0,069 . V M-0,114
$$

onde $A l b$ é o albedo do solo (300 a $2800 \mathrm{~nm}$ ) e $V M$ é o valor Munsell.

Os solos predominantes na bacia são Cambissolos, Gleissolos e Argissolos. A maior área da bacia, 42,64\% é formada por Cambissolo Háplico alumínico típico. Em seguida com 32,91\% tem-se a formação Argissolo Vermelho-Amarelo alumínico típico. Completam os tipos de solos dispostos na bacia hidrográfica do ribeirão Concórdia: Cambissolo Háplico Ta distrófico típico (17,44\%); Cambissolo Húmico alumínico típico (6,09\%) e Gleissolo Háplico Ta alumínico típico (0,92\%). Essa classificação foi realizada por Depiné (2010) baseada em EMBRAPA (2004).

A região climática onde a bacia do Ribeirão Concórdia está localizada, de acordo com a classificação de Thornthwaite é definida como sendo clima Mesotérmico Úmido, sem estação seca definida e com precipitações anuais entre 1600 a 1800 mm. De acordo com Köeppen, o clima é do tipo Cfa.

A vegetação original da área pertence à Floresta Ombrófila Densa. A distribuição espacial do uso do solo da bacia, foi determinada com uma imagem de satélite SPOT 5 com resolução espacial de $10 \mathrm{~m}$ em quatro bandas espectrais por Pinheiro et al. (2008), e é apresentada na figura1.

Conforme mostrado na figura 1 , a distribuiçao atual do uso e acupação do solo da bacia corresponde a Agricultura (hortaliças e feijão) (7,15\%), Pastagem (19,94\%), Reflorestamento (11,29\%), Fumo (0,60\%), Milho (9,83\%), Floresta Nativa (47,04\%) e Água (4,15\%).

\subsection{Modelo SWAT}

O modelo SWAT - Soil and Water Assessment Tool é um modelo de base física, com parâmetros distribuídos e simulação contínua. Ele é de domínio público e foi desenvolvido, para auxiliar no planejamento da

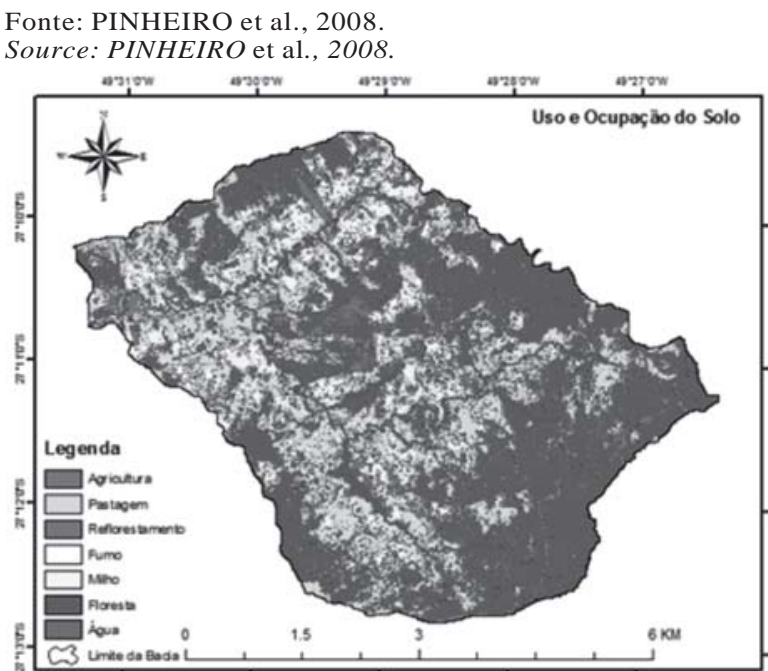

Figura 1 - Uso e ocupação do solo na Bacia do Ribeirão Concórdia em Santa Catarina, Brasil (2008).

Figure 1 - Landuse in the Concórdia river basin in Santa Catarina, Brazil (2008).

Revista Árvore, Viçosa-MG, v.37, n.5, p.859-869, 2013 
conservação do solo e da água, pelo Departamento de Agricultura norte-americano USDA, Agricultural Research Service (ARS), em Temple, no estado do Texas. Ele permite simular diferentes processos físicos do ciclo hidrológico na bacia hidrográfica (evapotranspiração, infiltração, escoamento superficial, subsuperficial e subterrâneo). Com o modelo SWAT pode-se simular hidrogramas e sedimentogramas e obter informações espaciais através da definição de cenários distintos (NEITSCH et al., 2005).

Para a utilização do modelo hidrológico SWAT são necessários dados de entrada, os quais são correlacionados com características físicas da bacia hidrográfica. Esses dados de entrada representam: o uso e ocupação das terras, parâmetros de solo (valores da massa específica aparente, teores de argila, silte e areia, condutividade hidráulica saturada, carbono orgânico e grupo hidrológico) o clima, o relevo e características hidrológicas da área de estudo.

Os dados são inseridos com o auxílio de uma interface gráfica do software GIS (ArcView ou Map Window), e são organizados em planos de informação cartográficos e dados tabulares. Os dados gráficos correspondem a mapas de uso do solo, mapas pedológicos, hidrografia e o modelo numérico do terreno (MNT). Os dados tabulares são os climáticos (velocidade do vento, precipitação, temperatura, radiação solar, umidade relativa do ar).

Basicamente o modelo SWAT considera a bacia dividida em sub-bacias com base no relevo, solos e uso da terra, preservando assim os parâmetros espacialmente distribuídos da bacia inteira e características homogêneas dentro da bacia. Essas sub-bacias são divididas em Unidades de Resposta Hidrológica (HRU's - Hydrological Response Units), que representam combinações únicas de uso do solo, tipo do solo e classe de rampa. As HRU's não interagem entre si. As respostas de cada HRU (escoamento, sedimentos e nutrientes) são calculadas separadamente e então somadas para encontrar-se a carga total da sub-bacia.

No modelo SWAT, a determinação da produção de água superficial da bacia hidrográfica é baseado no balanço hídrico, calculado em cada intervalo de tempo. O balanço hídrico na bacia é expresso por:

$$
S w_{t}=S w_{o}+\sum_{i=1}^{t}\left(P_{i}-Q_{i}-E T_{i}-\operatorname{Perc}_{i}-Q R_{i}\right)
$$

onde $\mathrm{Sw}_{\mathrm{t}}$ é oarmazenamento final de água no solo, $\mathrm{Sw}_{0}$ é o armazenamento inicial de água no solo (mm), t é o tempo (dias), $\mathrm{P}_{\mathrm{i}}$ é a precipitação $(\mathrm{mm}), \mathrm{Q}_{\mathrm{i}}$ é o

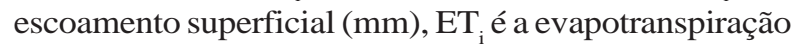
(mm), Perc $c_{\mathrm{i}}$ é a percolação (mm), e $\mathrm{QR}_{\mathrm{i}}$ é o fluxo de retorno (mm). As condições iniciais são geradas durante o período de aquecimento do modelo, que foi realizado com as simulações do período entre 2001 e 2006.

O modelo SWAT calcula a evaporação de solos e transpiração das plantas separadamente. A evaporação potencial da água do solo e a transpiração das plantas são funções da evapotranspiração potencial e do índice de área foliar. Três métodos são disponibilizados para o cálculo da evapotranspiração potencial: Hargreaves, Priestley-Taylor e Penman-Monteith. O método escolhido para o cálculo foi o de Priestley-Taylor (1972), por apresentar melhor adaptação aos dados meteorológicos disponíveis. Ele requer dados de radiação solar, temperatura, umidade relativa do ar, sendo expresso pela seguinte equação:

$$
E T P=K_{c}\left(\alpha \cdot \frac{\Delta}{\Delta+\gamma} \cdot E_{r}\right)
$$

onde ETP é a evapotranspiração potencial ( $\mathrm{mm} \mathrm{dia}^{-1}$ ), $\mathrm{E}_{\mathrm{r}}$ é a evaporação calculada pelo método do balanço de energia (mm/dia), $\Delta$ é a inclinação da curva de pressão de saturação de vapor em função da temperatura $\left(\mathrm{Pa} /{ }^{\circ} \mathrm{C}\right)$, g é a constante psicrométrica $\left(\mathrm{Pa} /{ }^{\circ} \mathrm{C}\right)$ e $\alpha$ é o parâmetro Priestley-Taylor. O valor utilizado é 1,26 , sendo representativo da média das diversas coberturas.

A partir da evapotranspiração potencial, o modelo SWAP calcula a evapotranspiração real levando-se em conta o estádio de desenvolvimento vegetal e o conteúdo de água no solo.

Para o cálculo do escoamento superficial, utilizou-se o método Curva Número (USDA-SCS, 1972), baseado no parâmetro CN que permite determinar a retenção superficial potencial. O valor de CN é função do grupo hidrológico e do uso e da ocupação do solo. O escoamento superficial é expresso por:

$$
Q=\frac{(P-0,2 S)^{2}}{P+0,8 S}
$$


onde Q é o escoamento superficial (mm), P é a precipitação $(\mathrm{mm})$ e $\mathrm{S}$ é a retenção potencial na superfície do solo $(\mathrm{mm})$. Esta expressão é valida para $\mathrm{P} \geq 0,2 \mathrm{~S}$.

A produção de sedimentos gerados pelo impacto da gota de chuva e pelo escoamento superficial é estimada para cada HRU através da Equação Universal de Perda de Solo Modificada (MUSLE). Ela é dada pela seguinte equação:

$$
S e d=11,8 .\left(Q . q p \cdot A_{H R U}\right)^{0,56} \text { K.C.PC.LS.CFRG }
$$

onde Sed é a produção de sedimentos em um dado dia (t), Q é o escoamento superficial ( $\mathrm{mm}$ ), qp é a vazão de pico $\left(\mathrm{m}^{3} \mathrm{~s}^{-1}\right), \mathrm{A}_{\mathrm{HRU}}$ é a área da HRU (ha), K é o fator de erodibilidade do solo, $C$ é o fator de manejo e cobertura do solo, Pc é o fator práticas conservacionistas, LS é o fator topográfico, CFRG é o fator de partículas grossas do solo.

\subsection{Calibração e validação do modelo}

A aplicação do modelo seguiu as etapas de simulação hidrológica descrita por Tucci (1998). Elas abrangem a calibração (ajuste) dos parâmetros do modelo a partir de séries de dados hidrológicos representativos de um período de interesse e a verificação dos parâmetros ajustados em outro período. A análise de eficiência da calibração e da verificação dos parâmetros do modelo é efetuada pelo uso de coeficientes estatísticos. Várias variáveis podem ser utilizadas nas etapas de calibração e verificação dos parâmetros do modelo hidrológico, como o ajuste dos balanços hídricos observados e simulados, hidrogramas observados e simulados e sedimentogramas observados e simulados.

O modelo SWAT foi calibrado comparando-se as séries de dados de vazão e de concentrações de sedimentos observadas e simuladas. Os dados observados utilizados foram obtidos das estações de monitoramento localizadas na bacia. O período utilizado para a calibração do modelo foi de janeiro de 2007 a dezembro de 2010 para escoamento e de novembro de 2008 a maio de 2009 para sedimentos. O período de validação do modelo compreende o período de janeiro de 2011 a dezembro de 2011. Ressalta-se que o modelo SWAT requer extensão das séries de dados para obtenção das condições iniciais. Assim, o modelo foi alimentado com séries de dados do período compreendido entre 2001 e 2010. Posteriormente, este período longo, foi utilizado para geração dos cenários de uso do solo na bacia.
Para o processo de calibração, inicialmente executou-se a análise automática de sensibilidade do modelo aos parâmetros. Os parâmetros que apresentam maior sensibilidade nos resultados do modelo foram alterados manualmente até se atingir a simulação adequada dos hidrogramas e sedimentograma, quando comparado aos valores medidos. Arnold et al. (2000) identificaram que o modelo SWAT é sensível a mais de 100 variáveis relacionadas à vegetação, manejo dado solo, solos, clima, aquífero, canal e reservatório.

Para avaliação do desempenho do ajuste do modelo foram utilizados os coeficientes de eficiência de NashSutcliffe (COE) e o de massa residual (CMR). Estes coeficientes são os mais utilizados para avaliação da eficiência de modelos hidrológicos. O COE é obtido pela expressão:

$$
C O E=1-\frac{\sum_{i=1}^{n}\left(E_{o b}-E_{c a l}\right)^{2}}{\sum_{i=1}^{n}\left(E_{o b}-E_{m}\right)^{2}}
$$

onde $E_{o b}$ é o valor observado, $E_{c a l}$ é o valor calculado, $E_{m}$ é a média da série de valores observados no período. O COE varia de infinito negativo até o valor máximo de 1 , que representa a eficiência máxima.

O coeficiente de massa residual (CMR) indica quando o modelo superestima (valores negativos) ou subestima (valores positivos) os valores simulados. Ele é expresso por:

$$
C M R=\frac{\sum_{i=1}^{n} E_{o b}-\sum_{i=1}^{n} E_{c a l}}{\sum_{i=1}^{n} E_{o b}}
$$

onde $E_{o b}$ é o valor observado e $E_{c a l}$ é o valor calculado.

\subsection{Cenários de uso do solo}

Após as etapas de calibração e de verificação do escoamento e da produção de sedimentono modelo SWAT, foram gerados e simulados os três cenários de uso do solo.

Os cenários simulados foram estabelecidos através da transformação do plano de informação gráficasdo uso e ocupação do solo. Em relação à classificação atual de uso e de ocupação do solo da bacia, 95,85\% da área foi alterada para o uso de solo considerado

Revista Árvore, Viçosa-MG, v.37, n.5, p.859-869, 2013 
no cenário. Somente $4,15 \%$ da área total da bacia, que correspondem à água, foi mantida em todos os cenários.

Assim, foram considerados os seguintes cenários:

Cenário 1:denominado cenário agricultura, considera que o uso na bacia é deagricultura anual, onde todas as culturas e manejos agrícolas são uniformes. No modelo SWAT esse uso de solo é Agricultural Land-Generic, o qual apresenta parâmetros médios específicos.

Cenário 2: denominado cenário mata nativa, considera que a bacia é ocupada pela Floresta SempreVerde com vegetação do tipo Ombrófila Densa.

Cenário 3: denominado cenário pastagem perene, sendo adotados os parâmetros dogênero Paspalun spp.

Todos os parâmetros utilizados para cada tipo de uso de solo (culturas) adotado foram os indicados nos manuais do modelo SWAT, apresentados por WINCHELL et al.(2010) e NEITSCHI et al. (2005).

Esses cenários de uso do soloforam avaliados e comparados com o cenário de uso e ocupação do solo atual da bacia do ribeirão Concórdia, para o período compreendido entre 2001 e 2010.

\section{RESULTADOS}

Na Figura 2 são apresentados os hidrogramas e os sedimentogramas observados e simulados, para os períodos de calibração do modelo SWAT. Na etapa de calibração do modelo SWAT obteve-se um coeficiente de Nash-Sutcliffe (COE) diário de 0,767 e mensal de 0,823 para a vazão. Para a produção de sedimento o COE diário foi de 0,308 e o mensal de 0,832 . O resultado do COE na verificação do modelo para vazão foi de 0,786 diário e 0,889 mensal e o CMR foi de -0,0921. Segundo Krysanova et al. (1998), para considerar um bom ajuste do modelo os valores COE devem estar entre 0,7 e 0,8. Conforme descrito anteriormente, tanto para vazão como para produção de sedimentos, os resultados de COE obtidos nas etapas de calibração e verificação foram superiores a 0,8 (mensal), representando assim um ajuste satisfatório do modelo SWAT para a bacia do ribeirão Concórdia.

O coeficiente de massa residual (CMR) obteve um valor de -0,0057 tanto diário como mensal, indicando que a diferença para a vazão foi praticamente nula na etapa de calibração do modelo. Isso indica que a quantidade de vazão simulada foi similar a observada. Para a produção de sedimentos, o CMR diário apresentou um valor satisfatório $(0,043)$, porém

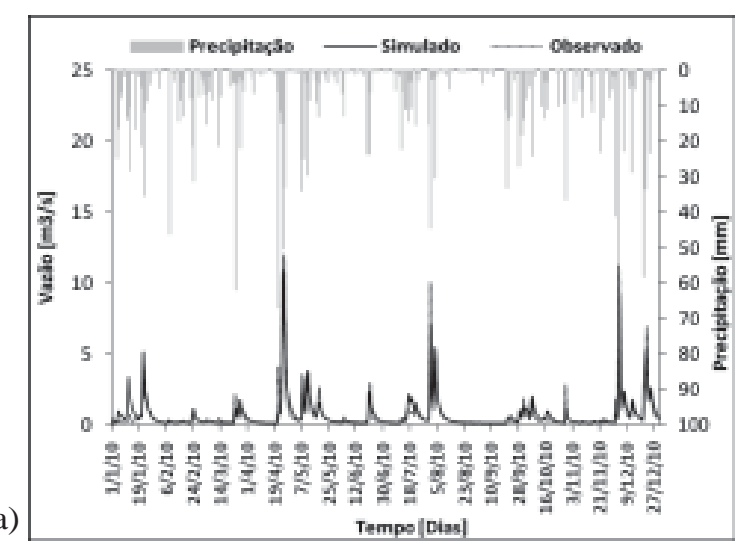

(a)

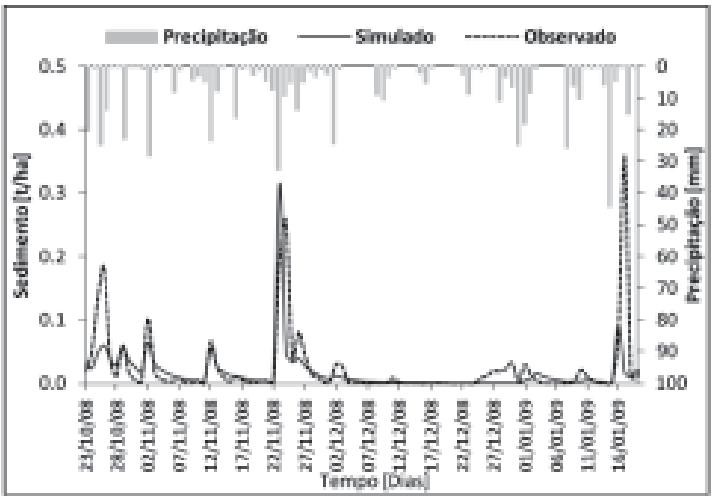

(b)

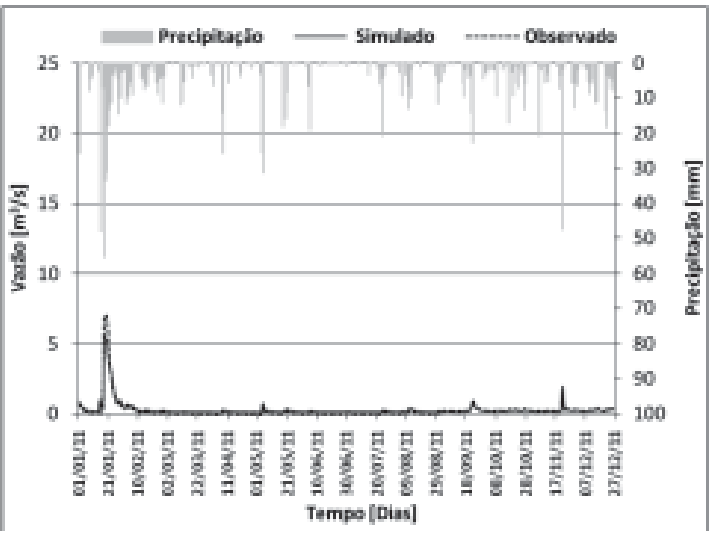

Figura 2 - Período de calibração: (a) vazões, (b) sedimentos e (c) período de validação das vazões da Bacia do Ribeirão Concórdia em Santa Catarina, Brasil.

Figure 2 - Calibration Period: (a) stream flows, (b) sediments and (c) validation period of stream flow, Concórdia river basin in Santa Catarina, Brazil. 
o CMR mensal $(0,275)$ indicou que a quantidade de sedimentos simulada ficou um pouco abaixo da observada.

No hidrograma das vazões observada se simuladas (Figura 2a) nota-se que o modelo simulou adequadamente tanto os valores mínimos, quanto os valores máximos.A comparação entre a produção diária de sedimento simulada e a observada (Figura 2b), mostra que o modelo reproduziu a distribuição e tendência das concentrações observadas, porém alguns valores elevados não foram adequadamente simulados. Apesar disso, como visto anteriormente, a produção de sedimentos também apresentou uma calibração satisfatória. O período de validação das vazões pode ser observado na Figura 2c.

Tanto os valores das vazões diárias, como da produção diária de sedimentos, apresentaram variações entre os cenários de uso simulados e o cenário atual de uso do solo. Os resultados das simulações para vazão e produção de sedimentos, dos quatro cenários e a comparação entre os mesmos e o uso atual são apresentados na Tabela 1. Os dados estão organizados em valores médios, máximos e mínimos diários, além da soma total para todo o período simulado, de 2001 a 2010.

A diferença da produção de sedimento entre esses cenários de uso e ocupação do solo pode ser observada também na Figura 4, que apresenta a distribuição média mensal para período estudado.

Todos esses resultados de vazão apresentados podem ser melhores avaliados quando se é analisado alguns dos componentes simulados pelo SWAT, no cálculo do ciclo hidrológico e do balanço hídrico. Na Tabela 2 são apresentados os principais parâmetros do balanço hídrico, gerados pelo modelo SWAT, para cada cenário gerado.

\section{DISCUSSÃO}

Na Tabela 1, fica evidente que o cenário pastagem apresentouum valor médio de vazão maior do que o cenário atual, enquanto o cenário mata nativa apresentou um valor menor, e o cenário agricultura gerou valor médio de vazão similar ao atual. As diferenças entre os cenários podem ser atribuídas às diferentes taxas de evapotranspiração das culturas estudadas e às alterações no escoamento superficial e infiltração de água no solo em função do tipo de sistema radicular, manejo e taxa de cobertura do solo.

Em comparação ao cenário atual de uso do solo, o cenário pastagem apresentou um acréscimo de cerca de 20\%. Mello et al., (2007) obtiveram um acréscimo no escoamento superficial de $4,5 \%$ com a simulação de um cenário composto por 100\% pastagem para uma microbacia hidrográfica na cidade de Lavras (MG).O solo usado para pastagem sofre com a compactação decorrente o pisoteio dos animais criando assim uma camada que pode reduzir a infiltração da água. Bertol et al. (2000) observaram que, com o aumento da oferta de forragem, a taxa de infiltração de água no solo diminui, em razão de uma maior pressão média dos animais sobre o solo. O cenário mata nativa, onde predominantemente a área foi transformada em floresta Ombrófila Densa, apresentouo maior decréscimo na vazão média, em relação ao cenário atual, cerca de $-8,70 \%$, isso devido, principalmente, a maior quantidade de matéria orgânica e ao aumento da evapotranspiração. Com maior matéria orgânica, o solo fica melhor estruturado e aumenta a infiltração de água no solo. Por outro lado, com a maior cobertura proporcionada pela floresta, ocorre maior interceptação da precipitação pluvial e menos água chega ao solo. Segundo Prusket al. (2003), quanto maior a evapotranspiração, menor será a umidade do

Tabela 1 - Comparação dos valores diários de vazão e sedimento no período de 2001 a 2010, da Bacia do Ribeirão Concórdia em Santa Catarina, Brasil.

Table 1 - Comparison of daily values of streamflow and sediment from 2001 to 2010, Concórdia river basin, Santa Catarina, Brazil.

\begin{tabular}{|c|c|c|c|c|c|c|c|c|}
\hline \multirow{2}{*}{ Cenários } & \multicolumn{4}{|c|}{ Vazão $\left(\mathrm{m}^{3} / \mathrm{s}\right)$} & \multicolumn{4}{|c|}{ Sedimento (t/ha/dia) } \\
\hline & Soma & Média & Mínimo & Máximo & Soma & Média & Mínimo & Máximo \\
\hline Atual & 1808,94 & 0,50 & $0,697 \times 10^{-4}$ & 31,75 & 922,30 & 0,26 & $3,51 \times 10^{-07}$ & 98,34 \\
\hline Agricultura & 1830,95 & 0,50 & $0,740 \times 10^{-4}$ & 32,09 & 2738,56 & 0,76 & $3,83 \times 10^{-08}$ & 302,57 \\
\hline Mata Nativa & 1688,49 & 0,46 & $0,716 \times 10^{-4}$ & 31,70 & 532,21 & 0,15 & $3,76 \times 10^{-08}$ & 57,88 \\
\hline Pastagem & 2235,44 & 0,61 & $0,738 \times 10^{-4}$ & 31,73 & 990,81 & 0,27 & $3,87 \times 10^{-08}$ & 89,21 \\
\hline
\end{tabular}


solo e, consequentemente, maior será a taxa de infiltração e menor o escoamento superficial. Esse resultado obtido corrobora com o trabalho desenvolvido por Lino (2009) na bacia Rio Negro (SC), onde foi observado que o maior impacto na redução do escoamento superficial foi obtido pela substituição do uso e cobertura atual do solo por mata nativa. Garcia et al. (2006) na bacia do rio Corumbataí (SP), também obtiveram uma redução na vazão média para o cenário mata nativa em relação ao cenário atual de uso de solo.

Os valores máximos de vazão não apresentaram uma diferença muito significativa entre os cenários simulados. O cenário agricultura foi o que gerou a vazão máxima mais elevado $\left(32,09 \mathrm{~m}^{-3} \mathrm{~s}^{-1}\right)$, cerca de $1,07 \%$ superior ao do uso atual. Entre as vazões mínimas, todos os cenários apresentaram valores superiores ao do uso atual, sendo que o cenário pastagem gerou um acréscimo de $5,7 \%$.

Os resultados da produção média diária de sedimento para o período de 2001 a 2010 (Tabela 1) mostram que os cenários agricultura e pastagem apresentaram valores superiores ao cenário atual. O cenário agricultura produziu a maior taxa de produção de sedimentos, 0,76 t/ha/ dia. Foi aproximadamente 3 vezes maior que o cenário atual, 0,26 t/ha/dia. Lino (2009) para a bacia do Rio Preto (SC) e Blainski et al., (2010b) para a bacia hidrográfica do Lajeado dos Fragosos (SC), apresentaram resultados nesse sentido, maiores valores médios na taxa de produção de sedimento com o cenário de uso de solo agricultura. A atividade agrícola altera a proteção dos solos, deixando-o susceptível a ação do impacto das gotas de chuva e ao escoamento superficial bem como pode promover a desestruturação da camada superficial do solo. A combinação destas ações proporciona o aumento da erosão do solo. O cenário mata nativa apresentou valor médio menor, em relação ao cenário uso atual. Isso se justifica pela diferença existente entre as culturas, como altura da planta, a camada de proteção no solo e diminuição da exposição do solo. Blainski et al., (2010a), com um cenário de reflorestamento com vegetação nativa, obtiveram menores valores de perda de solo em suas simulações. Para os valores máximos, o cenário que apresentou o valor mais elevado foi o da agricultura.

Em relação aos valores mínimos, o cenário que apresentou o menor valor foi à mata nativa, justificado pela maior e melhor cobertura e proteção do solo que evita assim os processos de erosão e de transporte de sedimentos. Segundo Larson et al. (1997), a rugosidade superficial é um importante fator na redução do escoamento superficial e, consequentemente, no transporte de sedimentos. A cobertura vegetal tem ação direta e efetiva na redução da erosão hídrica, devido á dissipação de energia cinética das gotas da chuva, diminuindo assim a desagregação das partículas do solo e o selamento superficial e aumenta a infiltração de água. A cobertura vegetal também reduz a velocidade do escoamento superficial e, consequentemente, a sua capacidade erosiva.

Conforme observado na Figura 3, o mês que apresentou os maiores valores de produção de sedimentos, para todos os cenários foi setembro, seguido por outubro. Isso pode ser explicado pela maior quantidade de chuvas que ocorrem nesses meses e pelas atividades agricolas desenvolvidas. O cenário agricultura apresentou os valores mais elevados para todos os meses, exceto para o mês de março, periodo durante o qual é realizada a colheita da maioria dos cultivos agrícolas e também é caracteristico por ser o período de estiagem.

Na Tabela 2 é possível observar que a precipitação de entrada foi à mesma para todos os cenários simulados. Os componentes da vazão fluvial, contribuição do escoamento superficial (S-Q), contribuição do fluxo lateral de vazão (L-Q) e, a água percolada além da zona de raiz (Perc) foram superiores para o cenário pastagem,

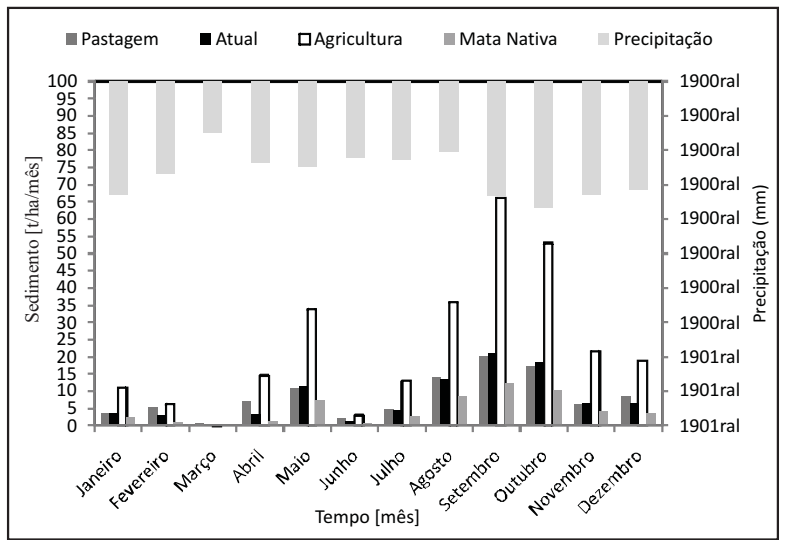

Figura 3 - Produção média mensal de sedimentos da Bacia do Ribeirão Concórdia em Santa Catarina, Brasil.

Figure 3 - Monthly sediments yields in the Concórdia river basin in Santa Catarina, Brazil. 
Tabela 2 - Valores médios diários das variáveis do balanço hídrico gerados pelo modelo SWAT, no período de 2001 a 2010, da Bacia do Ribeirão Concórdia em Santa Catarina, Brasil.

Table 2 - Daily average values of the water balance variables generated by the SWAT model from 2001 to 2010, Concórdia river basin, Santa Catarina, Brazil.

\begin{tabular}{ccccc}
\hline \multirow{2}{*}{ Variáveis } & \multicolumn{4}{c}{ Cenários } \\
\cline { 2 - 5 } & Atual & Agricultura & Mata Nativa & Pastagem \\
\hline P & 4,42 & 4,42 & 4,42 & 4,42 \\
Q & 1,53 & 1,51 & 1,39 & 1,86 \\
S-Q & 0,35 & 0,31 & 0,32 & 0,38 \\
L-Q & 1,04 & 1,10 & 1,00 & 1,35 \\
G-Q & 0,14 & 0,10 & 0,08 & 0,13 \\
Perc & 0,75 & 0,64 & 0,58 & 0,77 \\
Sw & 1021,4 & 1022,9 & 1014,1 & 1031,9 \\
E T & 2,30 & 2,36 & 2,51 & 1,94 \\
ETP & 3,06 & 3,06 & 3,06 & 3,06 \\
\hline
\end{tabular}

onde: P: precipitação (mm); Q: escoamento fluvial (mm); S-Q:contribuição do escoamento superficial (mm); L-Q: contribuição do fluxo lateral de vazão (mm); G-Q: contribuição das águas subterrâneas a vazão ( $\mathrm{mm} / \mathrm{dia})$; Perc: água que percola além da zona de raiz $(\mathrm{mm})$; Sw: quantidade de água no solo (mm); ETP: evapotranspiração potencial (mm); ET: evapotranspiração real (mm).

justificando assim os resultados dos valores mais altos de vazão obtidos. Em contra partida a evapotranspiração real (ET) foi inferior para o cenário pastagem. Deste modo, nota-se que quanto menor a evapotranspiração maior a vazão.A evapotranspiração reduz o volume de água armazenada no solo, responsável pela alimentação dos sistemas de drenagem superficial. Além disto, durante eventos de chuvas intensas, a redução do volume de água armazenada no solo, proporciona o aumento da taxa de infiltração, resultando na redução do escoamento superficial. Assim, ao contrário, a redução da evapotranspiração aumenta o armazenamento de água no solo, com maior alimentação dos rios e redução da taxa de infiltração, com aumento do escoamento superficial.

\section{CONCLUSÕES}

A aplicação do modelo hidrológico SWAT (Soil and Water Assessment Tool) na bacia hidrografica do ribeirão Concórdia, municipio de Lontras (SC), para a análise de vazão e sedimentos, permite estabelecer as seguintes conclusões:

- O modelo foi eficientemente calibrado e mostrou-se apto para as simulações dos cenários propostos;
- O cenário pastagem apresentou a maior vazão média diária e o cenário mata nativa apresentou a menor;

- Os valores máximos diários de vazão não apresentaram uma diferença muito significativa entre os cenários de uso do solo simulados;

- O cenário agricultura produziu a maior taxa de produção de sedimentos e o cenário mata nativa apresentou o menor valor médio;

- O tipo de uso, ocupação e cobertura do solo, interfere nos processos hidrológicos, consequentemente no regime de vazões e na produção de sedimentos em uma bacia hidrográfica.

- Em curto prazo, grandes alterações no uso e ocupação do solo acarretaram maiores impactos no regime e na disponibilidade hídrica da bacia.

\section{AGRADECIMENTOS}

AgradecemosaoMCT/FINEP/AÇÃOTRANSVERSAL - Previsão de Clima e Tempo 04/2008, convênio 1406/ 08, projeto 01.08.0568.00 pelo financiamento da pesquisa e a CAPES pela bolsa de mestrado.

\section{REFERÊNCIAS}

ARNOLD, J.G. et al.Regional estimation of base flow and groundwater recharge in Upper Mississippi river basin. Journal of Hydrology, v.227, n.1, p.21-40, 2000.

BERTOL, I. et al.Propriedades físicas do solo relacionadas a diferentes níveis de oferta de forragem capim elefante anão cv. Mott.

Pesquisa Agropecuária Brasileira, v.35, n.5, p.1047-1054, 2000.

BLAINSKI, E.; ARAUJO, I. S.; GOMES, P. Modelagem e simulação do uso do solo e as alterações no ambiente.In:CONGRESSO BRASILEIRO DE CIÊNCIA DO SOLO, 33., 2010, Uberlândia.Anais... Uberlândia: 2010a.

BLAINSKI, E.; GARBOSSA, L. H. P.; MALUTTA, S. Aplicação do modelo hidrológico SWAT (Soil and Water Assessment Tool) para a simulação da perda de solo e da disponibilidade hídrica em uma bacia hidrográfica não instrumentada. In: SIMPÓSIO DE RECURSOS HÍDRICOS DO NORDESTE,10., 2010, Fortaleza. Anais... Fortaleza: 2010b.

Revista Árvore, Viçosa-MG, v.37, n.5, p.859-869, 2013 
BLAINSKI, E. et al. Simulação de cenários de uso do solo na bacia hidrográfica do rio Araranguá utilizando a técnica da modelagem hidrológica. Revista Agropecuária Catarinense, v.24, n.1, mar. 2011.

BONNET, B. R. P.; FERREIRA, L. G.; LOBO, F. C.Relações entre qualidade da água e uso do solo em Goiás: uma análise à escala da bacia hidrográfica.Revista Árvore,v.32, n.2, p.311-322, 2008.

\section{DEPINÉ, H. Estudo do processo} sedimentológico na bacia experimental do Ribeirão Concórdia - SC.2010. 115f. Dissertação (Mestrado em Engenharia Ambiental) - Fundação Universidade Regional de Blumenau, Blumenau, 2010.

\section{EMPRESA BRASILEIRA DE PESQUISA} AGROPECUÁRIA - EMBRAPA. Solos do Estado de Santa Catarina. Rio de Janeiro: 2004. 726p. (Boletim de Pesquisa e Desenvolvimento, 46).

FOOD AND AGRICULTURE ORGANIZATION FOR THE UNITED NATIONS -FAO. 1999. Sistema de Informacion Geografica en el desarrollo sostenible. Disponivel em: $<$ http:// www.fao.org>. Acesso em: 9 jan. 2012.

GARCIA, G.J. et al. Impacto do uso da terra na erosão do solo e no balaço e qualidade de água na bacia do Rio Corumbataí - SP.

HOLOS Environment, v.6, n.2, p.118137, 2006.

KRYSANOVA, V.; MULLER-WOHLFEIL. D.; BECKER, A. Development and test of spatially distributed hydrological water quality model for mesoscale watersheds. Ecological Modeling, v.106, n.2, p.261-289, 1998.

KUHNLE, R. A. et al. Effect of land use changes on sediment transport. Water Research, v.32, NUMERO, p.3189-3196, 1996.

LARSON, W.E.; LINDSTROM, M.J.; SCHUMACHER, T.E. The role of severe storms in soil erosion: a problem needing consideration. Soil Water

Conservation,v.52,n.1, p.90-95, 1997.
LELIS, T. A.; CALIJURI, M. L. Modelagem hidrossedimentológica de bacia hidrográfica na região sudeste do Brasil, utilizando o SWAT. Revista Ambiente \&Água, v.5, n.2, p.158-174, 2010.

LINO, J. L. F. Análise da dinâmica hidrossedimentológica da Bacia Hidrográfica do Rio Preto (SC) com o modelo SWAT. 2009. 150f. Dissertação (Mestrado em Engenharia Ambiental)Universidade Federal de Santa Catarina, Florianópolis, 2009.

MACHADO R. E. ; VETTORAZZI C. A. ; XAVIER A. C. Simulação de Cenários Alternativos de Uso da Terra em uma Microbacia Utilizando Técnicas de Modelagem e Geoprocessamento. Revista Brasileira de Ciências do Solo, v. 27, p. 727-733, 2003.

MELLO, R. C.; LIMA, M.J.; SILVA, M.A. Simulação do deflúvio e vazão de pico em microbaciahidrográfica com escoamento efêmero. Revista Brasileira de Engenharia Agrícola e Ambiental,v.11, n.4, p.410-419, 2007.

NEITSCH, S. L. et al. Soil and water assessment tool: Theoretical documentation - Version 2005. Temple: Agricultural Research Service (USDA) e Texas Agricultural Experiment Station (Texas AeM University). 2005. 541p.

PARANHOS, R. M.; SARTORI, A.; PAIVA, J. B. D. Amostrador de sedimentos em suspensão de nível descendente - Desenvolvimento e avaliação. In Anais $5^{\circ}$. Encontro Nacional de Engenharia de Sedimentos, São Paulo, 2002. pp.139-146.

PINHEIRO, A. et al. Relação entre uso da terra da faixa ciliar e a qualidade das águas em uma bacia agrícola. In:CONGRESO INTERAMERICANO DE INGENIERÍA SANITARIA Y AMBIENTAL DE AIDIS, 31., 2008, Santiago.Anais... Santiago: 2008.

PINHEIRO, A. et al.Uso do Solo na zona ripária de bacias agrícolas de pequeno a médioporte. Revista Árvore, v.35, n.6, p. 12451251, 2011. 
POST, D.P. et al.Predicting soil albedo from soil color and spectral reflectance data. Soil Science Society of America Journal, v.64, n.3, p.1027-1234, 2000.

PRIESTLEY, C. H. B.; TAYLOR, R. J. On the assessment of surface heat flux and evaporation using large-scale parameters. Monthly Weather Revview, n.100, p.81-92, 1972.

PRUSKI, F. F.; RODRIGUES, L. N.; SILVA, D. D. Modelo hidrológico para estimativa do escoamento superficial em áreas agrícolas.

Revista Brasileira de Engenharia Agrícola e Ambiental, v.5, n.2, p.301-307, 2001.

REICHERT, J.M.;SUZUKI, L.E.A.S.; REINER, D.J. Compactação de solo em sistemas agropecuários e florestais: identificação, efeitos limites críticos e mitigação. In: CERETTA, C.A.; SILVA, L.S.; REICHERT, J.M. Tópicos em ciência do solo. Viçosa, MG: Sociedade Brasileira de Ciência do Solo, 2007. v.5. p.49-134.
SANTOS, L. L. Modelos hidráulicos-hidrológicos: conceitos e aplicações. Revista Brasileira de Geografia Física,v.2, n.3, p.1-19, 2009.

TEIXEIRA, L. P. Caracterização hidrológica dos solos da bacia experimental do ribeirão Concórdia. Blumenau, 2008. 124 p. Dissertação (Mestrado em Engenharia Ambiental), Fundação Universidade Regional de Blumenau.

TUCCI, C. E. M. Modelos hidrológicos. Porto Alegre: Universidade/UFRGS/Associação Brasileira de Recursos Hídricos, 1998.

TUCCI, C. E. M. Impactos da variabilidade climática e uso do solo sobre os recursos hídricos. Brasília: Fórum Brasileiro de Mudanças Climáticas, 2002. 150p.

USDA SOIL CONSERVATION SERVICE. National Engineering Handbook Section 4 Hydrology. Washington: 1972. Chapters 4-10.

WINCHELL, M. et al.ArcSwat Interface For SWAT 2009 - User's guide.Version 2009. 2010. 
